\title{
Theory of spontaneous emission factor and noise figure of optical parametric amplifiers
}

This paper was downloaded from TechRxiv (https://www.techrxiv.org).

\section{LICENSE}

CC BY 4.0

SUBMISSION DATE / POSTED DATE

$16-01-2022$ / 21-01-2022

\section{CITATION}

Kikuchi, Kazuro (2022): Theory of spontaneous emission factor and noise figure of optical parametric amplifiers. TechRxiv. Preprint. https://doi.org/10.36227/techrxiv.18489533.v1

$\mathrm{DOI}$

10.36227/techrxiv.18489533.v1 


\title{
Theory of spontaneous emission factor and noise figure of optical parametric amplifiers
}

\author{
Kazuro Kikuchi \\ National Institution for Academic Degrees and \\ Quality Enhancement of Higher Education
}

\begin{abstract}
This study develops a theory of noise in optical parametric amplifiers (OPAs) based on the quantum-mechanical Langevin equations. We derive theoretical expressions for the spontaneous emission factor of OPAs for the first time. This enables analyzing the effect of internal loss on the noise characteristics. In addition, we analyze the noise figures (NFs) of the laser amplifier, phase-insensitive OPA, and phase-sensitive OPA. The NFs in the direct-detection and coherent systems are compared, and the low-noise characteristics of OPAs are theoretically demonstrated under the influence of internal loss.
\end{abstract}

\section{Introduction}

Laser amplifiers, such as erbium-doped fiber amplifiers and semiconductor optical amplifiers, have widely been introduced in practical optical communication systems and devices. The noise characteristics of laser amplifiers are critical for improving the performance of systems and devices. Theoretically, laser-amplifier noise has always been analyzed using a master-equation approach [1], which focuses on fluctuations in the number of photons. The spontaneous emission factor, determined from the balance between gain and loss of laser amplifiers, plays an essential role in describing the noise characteristics.

Recently, phase-insensitive optical parametric amplifiers (OPAs) were experimentally studied in wavelength-division-multiplexed coherent optical transmission systems [2]. Their low-noise wideband characteristics are suitable for large-capacity long-distance optical transmission systems. Moreover, in principle, phase-sensitive OPAs can perform noise-free optical amplification, although they experience considerably more technical difficulties than phase-insensitive OPAs [3]. Despite these advantages, there are few theoretical reports on how the noise characteristics of OPAs are influenced by their internal loss, which cannot be neglected in practical devices [4].

This study develops a theory of noise in OPAs to analyze the effect of internal loss of OPAs on their noise characteristics. We use the quantum-mechanical Langevin-equation approach to derive the theoretical expressions for the spontaneous emission factor of phase-sensitive and phase-insensitive OPAs, respectively. To the best of our knowledge, this is the first study that derives such theoretical expressions. Using the derived spontaneous emission factor, the noise characteristics of OPAs can be analyzed in a simple manner.

Next, we theoretically compare the noise figures (NFs) of a laser amplifier, phase-insensitive OPA, and phase-sensitive OPA. The NFs of a laser amplifier and a phase-insensitive OPA in direct-detection (DD) systems were compared when the number of photons of an output signal was measured. Meanwhile, the NFs of a laser amplifier, a phase-insensitive OPA, and a phase-sensitive OPA in coherent systems were compared when the in-phase component (i.e., the real part) of the electric field of an output signal is measured. The NFs are numerically calculated as a function of gross gain of the amplifiers, and the low-noise characteristics of OPAs are clearly demonstrated, even under the influence of internal loss. 
The organization of this paper is as follows. Section 2 summarizes the theory of noise in laser amplifiers based on the quantum-mechanical Langevin equation. After elucidating properties of fluctuation operators associated with gain and loss, we derive a closed-form formula for the amplified electric field including noise, with which the noise characteristics are re-examined. In Sec. 3, we develop a theory of noise in phase-insensitive OPAs, following the theory of laser amplifiers in Sec. 2. By deriving the spontaneous emission factor, we obtain a closed-form expression for the amplified electric field that enables simple analyses of their noise characteristics. Section 4 extends the theory of noise in phase-insensitive OPAs to phase-sensitive OPAs. In Sec. 5, we theoretically analyze the NFs of a laser amplifier, phase-insensitive OPA, and phase-sensitive OPA. The NFs in the DD and coherent systems are compared with each other. Section 6 concludes this paper.

\section{Laser amplifiers}

\subsection{Langevin equation for laser amplifiers and its solution}

When the annihilation operator for a signal electric field, $\hat{a}_{\ell}$, passes through a medium with gain and loss, it obeys the following Langevin equation [5]:

$$
\frac{d \hat{a}_{\ell}(t)}{d t}=\frac{1}{2}\left(g_{\ell}-\gamma\right) \hat{a}_{\ell}(t)+\hat{f}_{g}(t)+\hat{f}_{\gamma}(t)
$$

where $g_{\ell}$ and $\gamma$ denote the gain and loss coefficients, respectively. The gain coefficient, $g_{\ell}$, is written as

$$
g_{\ell}=A N_{2}
$$

where $A$ represents Einstein's $A$ coefficient, and $N_{2}$ is the density of atoms excited into the upper laser level. Meanwhile, the loss coefficient, $\gamma$, is expressed as

$$
\gamma=A N_{1}+\gamma_{w}
$$

where $N_{1}$ is the density of atoms at the lower laser level, and $\gamma_{w}$ represents the loss coefficient of the waveguide. The fluctuation operators, $\hat{f}_{\gamma}$ and $\hat{f}_{g}$, result from the loss and gain of the medium, respectively. The solution to Eq. (1) is given as

$$
\hat{a}_{\ell}(t)=\exp \left(\frac{\left(g_{\ell}-\gamma\right) t}{2}\right)\left[\hat{a}_{\ell}(0)+\int_{0}^{t}\left(\hat{f}_{g}\left(t^{\prime}\right)+\hat{f}_{\gamma}\left(t^{\prime}\right)\right) \exp \left(-\frac{\left(g_{\ell}-\gamma\right) t^{\prime}}{2}\right) d t^{\prime}\right] .
$$

\subsection{Properties of the fluctuation operators in laser amplifiers}

The annihilation operator, $\hat{a}_{\ell}(t)$, and the creation operator, $\hat{a}_{\ell}^{\dagger}(t)$, must satisfy the commutation relation given by

$$
\left[\hat{a}_{\ell}(t), \hat{a}_{\ell}^{\dagger}(t)\right]=1
$$

Consequently, Eqs. (4) and (5) demonstrate that the commutation relation between $\hat{f}_{\gamma}(t)$ and $\hat{f}_{\gamma}^{\dagger}\left(t^{\prime}\right)$, and that between $\hat{f}_{g}(t)$ and $\hat{f}_{g}^{\dagger}\left(t^{\prime}\right)$, should be given [6] by

$$
\begin{aligned}
& {\left[f_{\gamma}(t), f_{\gamma}^{\dagger}\left(t^{\prime}\right)\right]=\gamma \delta\left(t-t^{\prime}\right),} \\
& {\left[f_{g}(t), f_{g}^{\dagger}\left(t^{\prime}\right)\right]=-g_{\ell} \delta\left(t-t^{\prime}\right) .}
\end{aligned}
$$


By contrast, according to the quantum damping theory [5], the correlation properties between the above-mentioned fluctuation operators are expressed, when the photon energy is much larger than the thermal energy, as

$$
\begin{aligned}
& \left\langle\hat{f}_{\gamma}^{\dagger}(t) \hat{f}_{\gamma}\left(t^{\prime}\right)\right\rangle=0 \\
& \left\langle\hat{f}_{\gamma}(t) \hat{f}_{\gamma}^{\dagger}\left(t^{\prime}\right)\right\rangle=\gamma \delta\left(t-t^{\prime}\right), \\
& \left\langle\hat{f}_{g}^{\dagger}(t) \hat{f}_{g}\left(t^{\prime}\right)\right\rangle=g_{\ell} \delta\left(t-t^{\prime}\right), \\
& \left\langle\hat{f}_{g}(t) \hat{f}_{g}^{\dagger}\left(t^{\prime}\right)\right\rangle=0 .
\end{aligned}
$$

In addition, $\hat{f}_{g}$ and $\hat{f}_{\gamma}$ have no correlation.

\subsection{Average number of output photons from laser amplifiers}

The photon-number operator of the output signal is given by $\hat{n}_{\ell}(t)=\hat{a}_{\ell}^{\dagger}(t) \hat{a}_{\ell}(t)$. We can obtain its average from Eq. (4) through Eqs. (8)-(11) as

$$
\left\langle\hat{n}_{\ell}(t)\right\rangle=G_{\ell} \Gamma\left\langle\hat{n}_{\ell}(0)\right\rangle+\left(G_{\ell} \Gamma-1\right) n_{s p, \ell},
$$

where the gross gain of the amplifier, $G_{\ell}$, the total loss, $\Gamma$, and the spontaneous emission factor, $n_{s p, \ell}$, are given by

$$
\begin{aligned}
G_{\ell} & =\exp \left(g_{\ell} t\right), \\
\Gamma & =\exp (-\gamma t), \\
n_{s p, \ell} & =\frac{g_{\ell}}{g_{\ell}-\gamma} .
\end{aligned}
$$

If the waveguide loss is negligible, $n_{s p, \ell}$ is written as

$$
n_{s p, \ell}=\frac{N_{2}}{N_{2}-N_{1}},
$$

which represents the degree of population inversion of lasing materials, as described in [1]. The first term on the right-hand side of Eq. (12) represents the average number of photons of the amplified signal, whereas the second term represents the amplified spontaneous emission (ASE).

\subsection{Closed-form expression of the electric field of the laser-amplifier output}

We convert Eq. (4) into a closed-form expression of the amplified electric field. Using the net gain, $G_{\ell} \Gamma$, and $n_{s p, \ell}$, we define the noise operator, $\hat{c}_{\ell}^{\dagger}$, as

$$
\sqrt{\left(G_{\ell} \Gamma-1\right) n_{s p, \ell}} \hat{c}_{\ell}^{\dagger}=\sqrt{G_{\ell} \Gamma} \int_{0}^{t} \hat{f}_{g}\left(t^{\prime}\right) \exp \left(-\frac{\left(g_{\ell}-\gamma\right) t^{\prime}}{2}\right) d t^{\prime} .
$$

Equations (7), (13)-(15), and (17) demonstrate that the noise operators, $\hat{c}_{\ell}$ and $\hat{c}_{\ell}^{\dagger}$, satisfy the following commutation relation:

$$
\left[\hat{c}_{\ell}, \hat{c}_{\ell}^{\dagger}\right]=1
$$

Equation (18) suggests that $\hat{c}_{\ell}$ and $\hat{c}_{\ell}^{\dagger}$ are the annihilation and creation operators for photons, respectively. Moreover, we define the noise operator, $\hat{d}_{\ell}$, as

$$
\sqrt{\left(G_{\ell} \Gamma-1\right)\left(n_{s p, \ell}-1\right)} \hat{d}_{\ell}=\sqrt{G_{\ell} \Gamma} \int_{0}^{t} \hat{f}_{\gamma}\left(t^{\prime}\right) \exp \left(-\frac{\left(g_{\ell}-\gamma\right) t^{\prime}}{2}\right) d t^{\prime} .
$$


Equations (6), (13)-(15), and (19) yield the commutation relation between $\hat{d}_{\ell}$ and $\hat{d}_{\ell}^{\dagger}$ as

$$
\left[\hat{d}_{\ell}, \hat{d}_{\ell}^{\dagger}\right]=1
$$

suggesting that $\hat{d}_{\ell}$ and $\hat{d}_{\ell}^{\dagger}$ are the annihilation and creation operators for photons, respectively. The noise operators, $\hat{c}_{\ell}$ and $\hat{d}_{\ell}$, are not correlated with each other, and have no correlation with the input signal, $\hat{a}_{\ell}(0)$.

Consequently, by using Eqs. (17) and (19), Eq. (4) is reduced to the new closed-form expression of the amplified electric field, given by

$$
\hat{a}_{\ell}(t)=\sqrt{G_{\ell} \Gamma} \hat{a}_{\ell}(0)+\sqrt{\left(G_{\ell} \Gamma-1\right) n_{s p, \ell}} \hat{c}_{\ell}^{\dagger}+\sqrt{\left(G_{\ell} \Gamma-1\right)\left(n_{s p, \ell}-1\right)} \hat{d}_{\ell} .
$$

The first term represents the electric field of the amplified signal, the second term represents noise associated with gain, and the third term represents noise associated with loss.

\subsection{Effects of loss and gain on amplifier noise}

This subsection examines the meaning of Eq. (21) in detail. We first consider the special case in which the amplifier has no gain, i.e., $g_{\ell}=0$, but has loss, i.e., $\gamma>0$. Given that $n_{s p, \ell}=0$ in such a case, Eq. (21) can be simplified as

$$
\hat{a}_{\ell}(t)=\sqrt{\Gamma} \hat{a}_{\ell}(0)+\sqrt{1-\Gamma} \hat{d}_{\ell},
$$

where the second term indicates that the loss-induced vacuum fluctuation merged into the signal. Next, we consider the case in which the amplifier has no loss, i.e., $\gamma=0$, but has gain, i.e., $g_{\ell}>0$. Noting that $n_{s p, \ell}=1$ in this case, we have

$$
\hat{a}_{\ell}(t)=\sqrt{G_{\ell}} \hat{a}_{\ell}(0)+\sqrt{G_{\ell}-1} \hat{c}_{\ell}^{\dagger},
$$

where the second term represents the ASE noise from the gain medium.

Equations (22) and (23) are well-known formulae describing the loss and gain effects, respectively, on an electric field [7]. However, the newly derived Eq. (21) generalizes them when loss and gain coexist.

\subsection{Noise formulae for laser amplifiers}

The noise characteristics of laser amplifiers can be calculated by simple algebraic manipulation of the creation and annihilation operators in Eq. (21). For example, the variance of the photon number is obtained from Eq. (21) after straightforward calculations as

$$
\begin{aligned}
\left\langle\Delta \hat{n}_{\ell}(t)^{2}\right\rangle & =\left(G_{\ell} \Gamma\right)^{2}\left(\left\langle\hat{n}_{\ell}(0)^{2}\right\rangle-\left\langle\hat{n}_{\ell}(0)\right\rangle^{2}-\left\langle\hat{n}_{\ell}(0)\right\rangle\right) \\
& +G_{\ell} \Gamma\left\langle\hat{n}_{\ell}(0)\right\rangle+\left(G_{\ell} \Gamma-1\right) n_{s p, \ell} \\
& +2 G_{\ell} \Gamma\left(G_{\ell} \Gamma-1\right)\left\langle\hat{n}_{\ell}(0)\right\rangle n_{s p, \ell}+\left(G_{\ell} \Gamma-1\right)^{2} n_{s p, \ell}^{2} .
\end{aligned}
$$

When the input light is in the coherent state, the first term on the right-hand side of Eq. (24) is zero. The second and third terms represent the shot noise caused by the signal and ASE, respectively; and the fourth and fifth terms represent the signal-ASE and ASE-ASE beat noise, respectively [8].

Moreover, the average and variance of the real part of the amplified electric field are given from Eq. (21) as

$$
\begin{aligned}
\left\langle\hat{a}_{\ell r}(t)\right\rangle & =\sqrt{G_{\ell} \Gamma}\left\langle\hat{a}_{\ell r}(0)\right\rangle, \\
\left\langle\Delta \hat{a}_{\ell r}(t)^{2}\right\rangle & =G_{\ell} \Gamma\left\langle\Delta \hat{a}_{\ell r}(0)^{2}\right\rangle+\left(G_{\ell} \Gamma-1\right) n_{s p, \ell}\left\langle\hat{c}_{\ell r}^{2}\right\rangle+\left(G_{\ell} \Gamma-1\right)\left(n_{s p, \ell}-1\right)\left\langle\hat{d}_{\ell r}^{2}\right\rangle,
\end{aligned}
$$


where the suffix $r$ of the operators indicates the real part. Noting that $\left\langle\Delta \hat{a}_{\ell r}(0)^{2}\right\rangle=1 / 4$, and $\left\langle\hat{c}_{\ell r}^{2}\right\rangle=\left\langle\hat{d}_{\ell r}^{2}\right\rangle=1 / 4$, we find from Eq. (26)

$$
\left\langle\Delta \hat{a}_{\ell r}(t)^{2}\right\rangle=\frac{1}{4}+\frac{1}{2}\left(G_{\ell} \Gamma-1\right) n_{s p, \ell} .
$$

The first term on the right-hand side of Eq. (27) originates from vacuum fluctuations, whereas the second term originates from ASE. The noise characteristics of the imaginary part of the signal electric field, $\hat{a}_{\ell i}(t)$, are the same as those of the real part, because laser amplifiers can amplify the real and imaginary parts of the signal electric field by the same gain, as expressed by Eq. (21).

\section{Phase-insensitive OPAs}

\subsection{Langevin equations for phase-insensitive OPAs and their solutions}

When a signal electric field and an idler electric field propagate through an OPA, the annihilation operator for the signal, $\hat{a}_{s}$, and the creation operator for the idler, $\hat{a}_{i}^{\dagger}$, satisfy the following Langevin equations [9]:

$$
\begin{aligned}
& \frac{d \hat{a}_{s}(t)}{d t}=-\frac{g_{p}}{2} \hat{a}_{i}^{\dagger}(t)-\frac{\gamma}{2} \hat{a}_{s}(t)+\hat{f}_{s}(t), \\
& \frac{d \hat{a}_{i}^{\dagger}(t)}{d t}=-\frac{g_{p}^{*}}{2} \hat{a}_{s}(t)-\frac{\gamma}{2} \hat{a}_{i}^{\dagger}(t)+\hat{f}_{i}^{\dagger}(t) .
\end{aligned}
$$

In these equations, the gain coefficient, $g_{p}$, is expressed as $g_{p}=\left|g_{p}\right| e^{i \phi}$, where $\left|g_{p}\right|$ is a real-numbered constant determined from the nonlinear optical coefficient and the pump power, and $\phi$ is determined from the phase difference between the signal and pump waves. In this study, we assume that the loss coefficients of the signal and idler have the same value of $\gamma$. Note that $\gamma$ of OPAs only includes the waveguide loss, in contrast to laser amplifiers (see Eq. (3)). The fluctuation operators, $\hat{f}_{s}$ and $\hat{f}_{i}$, result from the loss of the signal and idler, respectively.

The analytical solutions to Eqs. (28) and (29) can be derived as

$$
\begin{aligned}
\hat{a}_{s}(t) & =\left[\hat{a}_{s}(0)+\hat{A}(t)\right] \cosh \left(\frac{\left|g_{p}\right| t}{2}\right) \exp \left(-\frac{\gamma t}{2}\right) \\
& -\left[\exp (i \phi) \hat{a}_{i}^{\dagger}(0)+\hat{B}(t)\right] \sinh \left(\frac{\left|g_{p}\right| t}{2}\right) \exp \left(-\frac{\gamma t}{2}\right), \\
\hat{a}_{i}^{\dagger}(t) & =\left[\hat{a}_{i}^{\dagger}(0)+\exp (-i \phi) \hat{B}(t)\right] \cosh \left(\frac{\left|g_{p}\right| t}{2}\right) \exp \left(-\frac{\gamma t}{2}\right) \\
& -\exp (-i \phi)\left[\hat{a}_{s}(0)+\hat{A}(t)\right] \sinh \left(\frac{\left|g_{p}\right| t}{2}\right) \exp \left(-\frac{\gamma t}{2}\right),
\end{aligned}
$$

where the operators, $\hat{A}(t)$ and $\hat{B}(t)$, are expressed as

$$
\begin{aligned}
& \hat{A}(t)=\int_{0}^{t}\left[\hat{f}_{s}\left(t^{\prime}\right) \exp \left(\frac{\gamma t^{\prime}}{2}\right) \cosh \left(\frac{\left|g_{p}\right| t^{\prime}}{2}\right)+\hat{f}_{i}^{\dagger}\left(t^{\prime}\right) \exp (i \phi) \exp \left(\frac{\gamma t^{\prime}}{2}\right) \sinh \left(\frac{\left|g_{p}\right| t^{\prime}}{2}\right)\right] d t^{\prime}, \\
& \hat{B}(t)=\int_{0}^{t}\left[\hat{f}_{i}^{\dagger}\left(t^{\prime}\right) \exp (i \phi) \exp \left(\frac{\gamma t^{\prime}}{2}\right) \cosh \left(\frac{\left|g_{p}\right| t^{\prime}}{2}\right)+\hat{f}_{s}\left(t^{\prime}\right) \exp \left(\frac{\gamma t^{\prime}}{2}\right) \sinh \left(\frac{\left|g_{p}\right| t^{\prime}}{2}\right)\right] d t^{\prime} .
\end{aligned}
$$




\subsection{Properties of fluctuation operators in OPAs}

Similar to Eq. (6), the commutation relation between $\hat{f}_{s}(t)$ and $\hat{f}_{s}^{\dagger}\left(t^{\prime}\right)$, and that between $\hat{f}_{i}(t)$ and $\hat{f}_{i}^{\dagger}\left(t^{\prime}\right)$ are given by

$$
\begin{aligned}
& {\left[f_{s}(t), f_{s}^{\dagger}\left(t^{\prime}\right)\right]=\gamma \delta\left(t-t^{\prime}\right),} \\
& {\left[f_{i}(t), f_{i}^{\dagger}\left(t^{\prime}\right)\right]=\gamma \delta\left(t-t^{\prime}\right) .}
\end{aligned}
$$

Moreover, the correlation relations between them are given by

$$
\begin{aligned}
& \left\langle\hat{f}_{s}(t) \hat{f}_{s}^{\dagger}\left(t^{\prime}\right)\right\rangle=\gamma \delta\left(t-t^{\prime}\right), \\
& \left\langle\hat{f}_{s}^{\dagger}(t) \hat{f}_{s}\left(t^{\prime}\right)\right\rangle=0, \\
& \left\langle\hat{f}_{i}(t) \hat{f}_{i}^{\dagger}\left(t^{\prime}\right)\right\rangle=\gamma \delta\left(t-t^{\prime}\right), \\
& \left\langle\hat{f}_{i}^{\dagger}(t) \hat{f}_{i}\left(t^{\prime}\right)\right\rangle=0 .
\end{aligned}
$$

The operators, $\hat{f}_{s}$ and $\hat{f}_{i}$, are not correlated with each other, and are correlated with neither $\hat{a}_{s}(0)$ nor $\hat{a}_{i}(0)$.

When the signal is incident on the OPA but the idler is not, the signal gain does not depend on $\phi$, as expressed by Eq. (30). This mode of operation is referred to as phase-insensitive parametric amplification. In the following, we assume that $\hat{a}_{i}(0)=\Delta \hat{a}_{i}(0)$, where $\Delta \hat{a}_{i}(0)$ represents the vacuum fluctuations in the idler channel. As shown by Eq. (30), the vacuum fluctuations in the idler channel merge into the signal channel and increase the signal noise.

\subsection{Average number of output photons from phase-insensitive OPAs}

Using Eqs. (36)-(39), we obtain the following averages for the operators $\hat{A}(t)$ and $\hat{B}(t)$ from Eqs. (32) and (33):

$$
\begin{aligned}
& \left\langle\hat{A}^{\dagger}(t) \hat{A}(t)\right\rangle=\frac{1}{4}\left[\frac{\exp \left(\left(\left|g_{p}\right|+\gamma\right) t\right)-1}{1+\frac{\left|g_{p}\right|}{\gamma}}+\frac{\exp \left(\left(-\left|g_{p}\right|+\gamma\right) t\right)-1}{1-\frac{\left|g_{p}\right|}{\gamma}}-2(\exp (\gamma t)-1)\right], \\
& \left\langle\hat{B}^{\dagger}(t) \hat{B}(t)\right\rangle=\frac{1}{4}\left[\frac{\exp \left(\left(\left|g_{p}\right|+\gamma\right) t\right)-1}{1+\frac{\left|g_{p}\right|}{\gamma}}+\frac{\exp \left(\left(-\left|g_{p}\right|+\gamma\right) t\right)-1}{1-\frac{\left|g_{p}\right|}{\gamma}}+2(\exp (\gamma t)-1)\right] \text {, } \\
& \left\langle\hat{A}^{\dagger}(t) \hat{B}(t)+\hat{B}^{\dagger}(t) \hat{A}(t)\right\rangle=\frac{1}{2}\left[\frac{\exp \left(\left(\left|g_{p}\right|+\gamma\right) t\right)-1}{1+\frac{\left|g_{p}\right|}{\gamma}}-\frac{\exp \left(\left(-\left|g_{p}\right|+\gamma\right) t\right)-1}{1-\frac{\left|g_{p}\right|}{\gamma}}\right] .
\end{aligned}
$$

By using Eqs. (30) and (40)-(42), the average number of output signal photons, $\left\langle\hat{n}_{s}(t)\right\rangle=\left\langle\hat{a}_{s}^{\dagger}(t) \hat{a}_{s}(t)\right\rangle$, can be derived after straightforward calculations as

$$
\left\langle\hat{n}_{s}(t)\right\rangle=G_{p} \Gamma\left\langle\hat{n}_{s}(0)\right\rangle+\left(G_{p} \Gamma-1\right) n_{s p, p},
$$

where the gross gain, $G_{p}$, is given by

$$
G_{p}=\cosh ^{2}\left(\frac{\left|g_{p}\right| t}{2}\right)
$$


and $n_{s p, p}$ is the spontaneous emission factor, calculated as

$$
\begin{aligned}
n_{s p, p} & =1+\frac{1}{G_{p} \Gamma-1}\left\{\frac{1}{4\left(1+\frac{\left|g_{p}\right|}{\gamma}\right)}\left[1-\Gamma\left(\sqrt{G_{p}}-\sqrt{G_{p}-1}\right)^{2}\right]\right. \\
& \left.+\frac{1}{4\left(1-\frac{\left|g_{p}\right|}{\gamma}\right)}\left[1-\Gamma\left(\sqrt{G_{p}}+\sqrt{G_{p}-1}\right)^{2}\right]+\frac{1}{2}(1-\Gamma)\right\} .
\end{aligned}
$$

Equation (43) has the same form as Eq. (12), but $n_{s p, \ell}, G_{\ell}$, and $\left\langle\hat{n}_{\ell}(0)\right\rangle$ in Eq. (12) are replaced by $n_{s p, p}, G_{p}$, and $\left\langle\hat{n}_{s}(0)\right\rangle$ in Eq. (43), respectively.

\subsection{Closed-form expression of the electric field of the phase-insensitive OPA output}

We convert Eq. (30) into a closed-form expression of the amplified electric field. Let the noise operators $\hat{c}_{p}^{\dagger}$ and $\hat{d}_{p}$ be defined as

$$
\begin{aligned}
\sqrt{\left(G_{p} \Gamma-1\right) n_{s p, p}} \hat{c}_{p}^{\dagger} & =-\sqrt{\left(G_{p}-1\right) \Gamma} \exp (i \phi)\left[\Delta \hat{a}_{i}^{\dagger}(0)+\int_{0}^{t} \hat{f}_{i}^{\dagger}\left(t^{\prime}\right) \exp \left(\frac{\gamma t^{\prime}}{2}\right) \cosh \left(\frac{\left|g_{p}\right| t^{\prime}}{2}\right) d t^{\prime}\right] \\
& +\sqrt{G_{p} \Gamma} \exp (i \phi) \int_{0}^{t} \hat{f}_{i}^{\dagger}\left(t^{\prime}\right) \exp \left(\frac{\gamma t^{\prime}}{2}\right) \sinh \left(\frac{\left|g_{p}\right| t^{\prime}}{2}\right) d t^{\prime}, \\
\sqrt{\left(G_{p} \Gamma-1\right)\left(n_{s p, p}-1\right)} \hat{d}_{p} & =\sqrt{G_{p} \Gamma} \int_{0}^{t} \hat{f}_{s}\left(t^{\prime}\right) \exp \left(\frac{\gamma t^{\prime}}{2}\right) \cosh \left(\frac{|g| t^{\prime}}{2}\right) \\
& -\sqrt{\left(G_{p}-1\right) \Gamma} \int_{0}^{t} \hat{f}_{s}\left(t^{\prime}\right) \exp \left(\frac{\gamma t^{\prime}}{2}\right) \sinh \left(\frac{\left|g_{p}\right| t^{\prime}}{2}\right) d t^{\prime} .
\end{aligned}
$$

Using Eqs. (34) and (35), we can demonstrate the following commutation relations:

$$
\begin{aligned}
{\left[\hat{c}_{p}, \hat{c}_{p}^{\dagger}\right] } & =1, \\
{\left[\hat{d}_{p}, \hat{d}_{p}^{\dagger}\right] } & =1 .
\end{aligned}
$$

Consequently, $\hat{c}_{p}$ and $\hat{d}_{p}$ are the annihilation operators, and $\hat{c}_{p}^{\dagger}$ and $\hat{d}_{p}^{\dagger}$ are the creation operators for photons. Finally, we can derive the following closed-form expression for the electric field of the OPA output, using Eqs. (30), (32), (33), (46), and (47):

$$
\hat{a}_{s}(t)=\sqrt{G_{p} \Gamma} \hat{a}_{s}(0)+\sqrt{\left(G_{p} \Gamma-1\right) n_{s p, p}} \hat{c}_{p}^{\dagger}+\sqrt{\left(G_{p} \Gamma-1\right)\left(n_{s p, p}-1\right)} \hat{d}_{p} .
$$

The first term in Eq. (50) represents the electric field of the amplified signal, the second term represents the noise stemming from vacuum fluctuations in the idler channel, and the third term represents the noise associated with signal loss.

\subsection{Noise formulae for phase-insensitive OPAs}

Using Eq. (50), we can calculate the noise characteristics of the phase-insensitive OPAs by simple algebraic manipulation of the creation and annihilation operators. Given that Eq. (50) has the same form as Eq. (21), the noise formulae, Eqs. (24) and (27), are still valid for OPAs. When the input 
signal is in a coherent state, replacing $G_{\ell}, n_{s p, \ell}$, and $\left\langle\hat{n}_{\ell}(0)\right\rangle$ in Eqs. (24) and (27) with $G_{p}, n_{s p, p}$, and $\left\langle\hat{n}_{s}(0)\right\rangle$, respectively, we have the noise formulae, given by

$$
\begin{aligned}
\left\langle\Delta n_{s}(t)^{2}\right\rangle & =G_{p} \Gamma\left\langle\hat{n}_{s}(0)\right\rangle+\left(G_{p} \Gamma-1\right) n_{s p, p} \\
& +2 G_{p} \Gamma\left(G_{p} \Gamma-1\right)\left\langle\hat{n}_{s}(0)\right\rangle n_{s p, p}+\left(G_{p} \Gamma-1\right)^{2} n_{s p, p}^{2}, \\
\left\langle\Delta \hat{a}_{s r}(t)^{2}\right\rangle & =\frac{1}{4}+\frac{1}{2}\left(G_{p} \Gamma-1\right) n_{s p, p} .
\end{aligned}
$$

Similar to laser amplifiers, the real and imaginary parts of the signal electric field have the same noise characteristics.

\section{Phase-sensitive OPA}

\subsection{Langevin equation for phase-sensitive OPAs and its solution}

When the signal and idler waves in OPAs are degenerated, the gain becomes dependent on $\phi$. This mode of operation is referred to as phase-sensitive parametric amplification. Equation (28) yields the following Langevin equation governing the phase-sensitive parametric amplification:

$$
\frac{d \hat{a}_{s}(t)}{d t}=-\frac{g_{p}}{2} \hat{a}_{s}^{\dagger}(t)-\frac{\gamma}{2} \hat{a}_{s}(t)+\hat{f}_{s}(t) .
$$

If $\phi=\pi$, the Langevin equations for the real part, $\hat{a}_{s r}(t)$, and the imaginary part, $\hat{a}_{s i}(t)$, of the signal electric field are given by

$$
\begin{aligned}
& \frac{d \hat{a}_{s r}(t)}{d t}=\frac{\left|g_{p}\right|}{2} \hat{a}_{s r}(t)-\frac{\gamma}{2} \hat{a}_{s r}(t)+\frac{1}{2}\left(\hat{f}_{s}(t)+\hat{f}_{s}^{\dagger}(t)\right), \\
& \frac{d \hat{a}_{s i}(t)}{d t}=-\frac{\left|g_{p}\right|}{2} \hat{a}_{s i}(t)-\frac{\gamma}{2} \hat{a}_{s i}(t)+\frac{1}{2 i}\left(\hat{f}_{s}(t)-\hat{f}_{s}^{\dagger}(t)\right) .
\end{aligned}
$$

The solution to Eq. (54) is given by

$$
\hat{a}_{s r}(t)=\exp \left(\frac{\left(\left|g_{p}\right|-\gamma\right) t}{2}\right)\left[\hat{a}_{s r}(0)+\frac{1}{2} \int_{0}^{t} \exp \left(-\frac{\left(\left|g_{p}\right|-\gamma\right) t^{\prime}}{2}\right)\left(\hat{f}_{s}\left(t^{\prime}\right)+\hat{f}_{s}^{\dagger}\left(t^{\prime}\right)\right) d t^{\prime}\right]
$$

whereas the solution to Eq. (55) is given by

$$
\hat{a}_{s i}(t)=\exp \left(-\frac{\left(\left|g_{p}\right|+\gamma\right) t}{2}\right)\left[\hat{a}_{s i}(0)+\frac{1}{2 i} \int_{0}^{t} \exp \left(\frac{\left(\left|g_{p}\right|+\gamma\right) t^{\prime}}{2}\right)\left(\hat{f}_{s}\left(t^{\prime}\right)-\hat{f}_{s}^{\dagger}\left(t^{\prime}\right)\right) d t^{\prime}\right] .
$$

Note that the real part of the signal electric field is amplified, whereas its imaginary part is deamplified, as expressed by

$$
\begin{aligned}
\left\langle\hat{a}_{s r}(t)\right\rangle & =\sqrt{G_{d} \Gamma}\left\langle\hat{a}_{s r}(0)\right\rangle, \\
\left\langle\hat{a}_{s i}(t)\right\rangle & =\sqrt{\frac{\Gamma}{G_{d}}}\left\langle\hat{a}_{s i}(0)\right\rangle,
\end{aligned}
$$

where the in-phase gross gain, $G_{d}$, is given by

$$
G_{d}=\exp \left(\left|g_{p}\right| t\right)
$$

Therefore, we can use only the real part of the signal electric field for coherent optical transmission systems. 


\subsection{Noise formulae for phase-sensitive OPAs}

In contrast with laser amplifiers and phase-insensitive OPAs, the second terms in Eqs. (56) and (57) cannot be written in terms of single annihilation operators for photons because of the phase-sensitive gain. Therefore, a closed-form expression for $\hat{a}_{s}(t)$ is not available, and the noise characteristics must be evaluated by performing integration in Eqs. (56) and (57). However, the second moment of the operators is not difficult to calculate. For example, the variance of the real part can be calculated, when the incident signal is in a coherent state, as

$$
\left\langle\Delta \hat{a}_{s r}^{2}(t)\right\rangle=\frac{1}{4}+\frac{\left(G_{d} \Gamma-1\right) n_{s p, d}}{4},
$$

where $n_{s p, d}$ represents the spontaneous emission factor, which is expressed as

$$
n_{s p, d}=\frac{\left|g_{p}\right|}{\left|g_{p}\right|-\gamma} .
$$

Meanwhile, the variance of the imaginary part is calculated as

$$
\left\langle\Delta \hat{a}_{s i}^{2}(t)\right\rangle=\frac{1}{4}+\frac{1}{4}\left(\frac{\Gamma}{G_{d}}-1\right) n_{s p, d}^{\prime},
$$

where the spontaneous emission factor, $n_{s p, d}^{\prime}$, is given as

$$
n_{s p, d}^{\prime}=\frac{\left|g_{p}\right|}{\left|g_{p}\right|+\gamma} .
$$

\section{$5 \quad$ NF of optical amplifiers}

\subsection{Definition of NF in DD systems}

The NF of amplifiers can be defined as the power ratio of the equivalent input noise to the actual input noise of the signal. The equivalent input noise of laser amplifiers in DD systems is given by $\left\langle\Delta \hat{n}_{\ell}(t)^{2}\right\rangle /\left(G_{\ell} \Gamma\right)^{2}$. The variance of the photon number of the input signal in the coherent state is given by $\left\langle\hat{n}_{\ell}(0)\right\rangle[8]$. Consequently, by using Eq. (24), the NF can be written as

$$
\mathrm{NF}_{D D 1, \ell}=\frac{G_{\ell} \Gamma n_{0} \cdot+\left(G_{\ell} \Gamma-1\right) n_{s p, \ell}+2 G_{\ell} \Gamma\left(G_{\ell} \Gamma-1\right) n_{s p, \ell} n_{0}+\left(G_{\ell} \Gamma-1\right)^{2} n_{s p, \ell}^{2}}{\left(G_{\ell} \Gamma\right)^{2} n_{0}},
$$

where $n_{0}=\left\langle\hat{n}_{\ell}(0)\right\rangle$. When $G_{\ell} \Gamma n_{0} \gg\left(G_{\ell} \Gamma-1\right) n_{s p, \ell}$, which means that the photon number of the amplified signal is much larger than that of the ASE, as is usually the case, and Eq. (65) is approximated as

$$
\mathrm{NF}_{D D 2, \ell}=\frac{1}{G_{\ell} \Gamma}+\frac{2\left(G_{\ell} \Gamma-1\right) n_{s p, \ell}}{G_{\ell} \Gamma} .
$$

If $G_{\ell} \Gamma \gg 1$, Eq. (66) is further reduced to $2 n_{s p, \ell}$, which is commonly called the NF of laser amplifiers [8]. Given that $n_{s p, \ell} \geq 1$ in the high-gain region, the lower limit of the NF is $2(3 \mathrm{~dB})$.

Similarly, in DD systems using phase-insensitive OPAs, the NF is given from Eq. (51) by

$$
\mathrm{NF}_{D D 1, p}=\frac{G_{p} \Gamma n_{0}+\left(G_{p} \Gamma-1\right) n_{s p, p}+2 G_{p} \Gamma\left(G_{p} \Gamma-1\right) n_{s p, p} n_{0}+\left(G_{p} \Gamma-1\right)^{2} n_{s p, p}^{2}}{\left(G_{p} \Gamma\right)^{2} n_{0}},
$$

where $n_{0}=\left\langle\hat{n}_{s}(0)\right\rangle$. When $G_{p} \Gamma n_{0} \gg\left(G_{p} \Gamma-1\right) n_{s p, p}$, Eq. (67) is approximated as

$$
\mathrm{NF}_{D D 2, p}=\frac{1}{G_{p} \Gamma}+\frac{2\left(G_{p} \Gamma-1\right) n_{s p, p}}{G_{p} \Gamma}
$$

In the high-gain region in which $G_{p} \Gamma \gg 1$, Eq. (68) approaches $2 n_{s p, p}$. Equation (45) shows that $n_{s p, p} \geq 1$; therefore, the lower limit of the NF is $2(3 \mathrm{~dB})$. 


\subsection{Numerical results on NF in DD systems}

Figures 1, 2, and 3 show the NFs of laser amplifiers and phase-insensitive OPAs in DD systems, calculated as a function of gross gain when $\Gamma=-3 \mathrm{~dB}, \Gamma=-5 \mathrm{~dB}$, and $\Gamma=-10 \mathrm{~dB}$, respectively. The red and black broken curves show rigorous $\mathrm{NFs}, \mathrm{NF}_{D D 1, \ell}$ and $\mathrm{NF}_{D D 1, p}$, respectively, when $n_{0}=100$. By contrast, the red and black solid curves show the approximated NFs, $\mathrm{NF}_{D D 2, \ell}$ and $\mathrm{NF}_{D D 2, p}$, respectively. Note that $\mathrm{NF}_{D D 2, \ell}$ and $\mathrm{NF}_{D D 2, p}$ are good approximations to $\mathrm{NF}_{D D 1, \ell}$ and $\mathrm{NF}_{D D 1, p}$, respectively. Moreover, $2 n_{s p, \ell}$ and $2 n_{s p, p}$ are represented by the red and black dash-dotted curves, respectively, when the gross gain is larger than $1 / \Gamma$. These parameters are effective only for the NF in the high-gain region.

Note that $\mathrm{NF}_{D D 1, p}$ is always smaller than $\mathrm{NF}_{D D 1, \ell}$ for prescribed values of the gross gain and $\Gamma$; this demonstrates the intrinsic low-noise characteristics of OPAs. Moreover, Figs. 1, 2, and 3 show how the noise characteristics of the laser amplifier and phase-insensitive OPA are deteriorated by their internal loss.

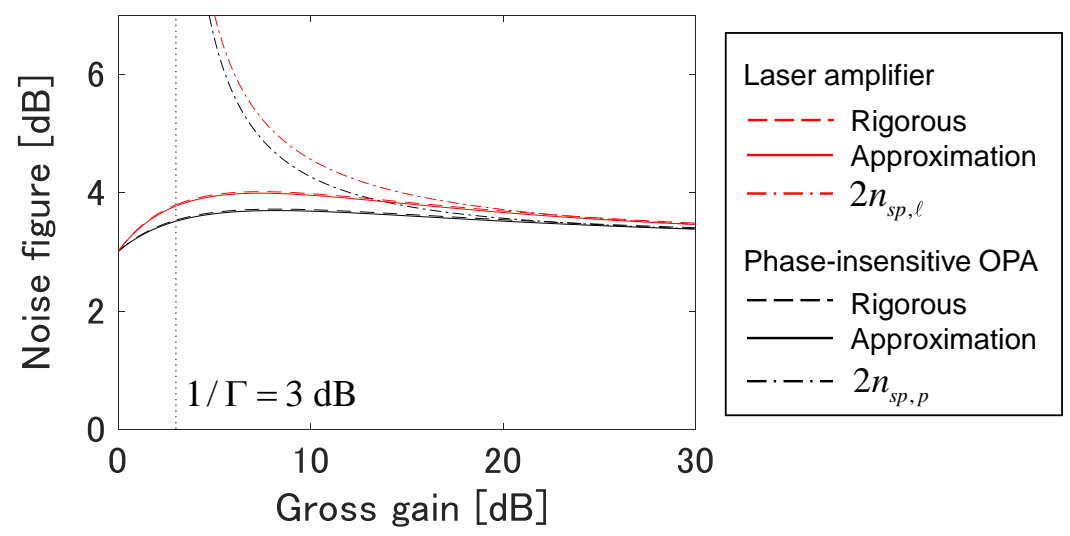

Figure 1: NFs of laser amplifiers and phase-insensitive OPAs in DD systems, calculated as the function of gross gain when $\Gamma=-3 \mathrm{~dB}$. Red and black broken curves: $\mathrm{NF}_{D D 1, \ell}$ and $\mathrm{NF}_{D D 1, p}$, respectively, when $n_{0}=100$. Red and black solid curves: $\mathrm{NF}_{D D 2, \ell}$ and $\mathrm{NF}_{D D 2, p}$, respectively. Red and black dash-dotted curves: $2 n_{s p, \ell}$ and $2 n_{s p, p}$, respectively.

\subsection{Definition of NF in coherent systems}

We assume that the real part (or imaginary part) of the signal electric field is measured by coherent receivers behind laser amplifiers. The equivalent input noise is given by $\left\langle\Delta \hat{a}_{\ell r}(t)^{2}\right\rangle /\left(G_{\ell} \Gamma\right)$. Meanwhile, the variance of the real part of the input signal electric field in the coherent state is equal to 1/4. Consequently, by using Eq. (27), the NF is written as

$$
\mathrm{NF}_{\operatorname{coh} 1, \ell}=\frac{1}{G_{\ell} \Gamma}+\frac{2\left(G_{\ell} \Gamma-1\right) n_{s p, \ell}}{G_{\ell} \Gamma},
$$

which is the same as the approximated NF for DD systems, given by Eq. (66). The measurement of the imaginary part of the signal electric field resulted in the same NF. Similarly, when phase-insensitive OPAs are employed, the NF is given as

$$
\mathrm{NF}_{c o h 1, p}=\frac{1}{G_{p} \Gamma}+\frac{2\left(G_{p} \Gamma-1\right) n_{s p, p}}{G_{p} \Gamma}
$$




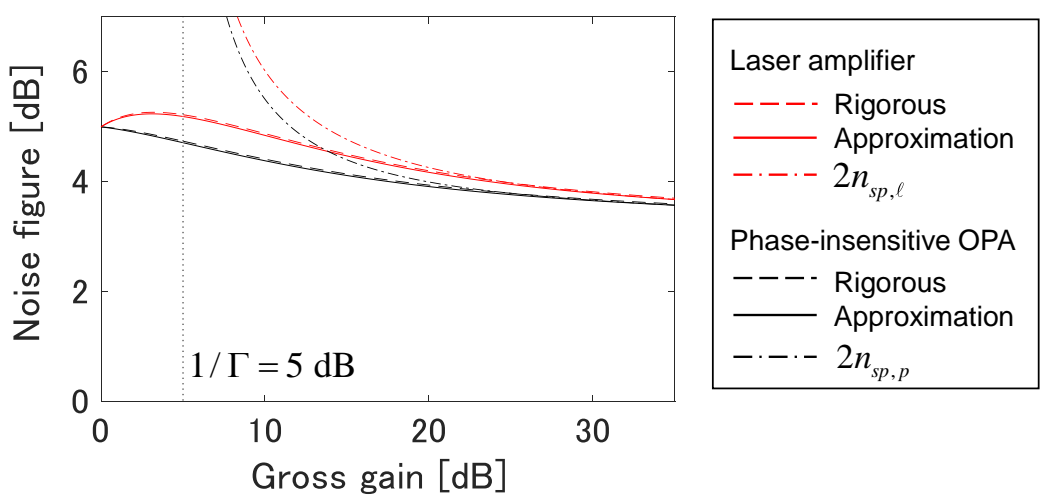

Figure 2: NFs of laser amplifiers and phase-insensitive OPAs in DD systems, calculated as a function of gross gain when $\Gamma=-5 \mathrm{~dB}$. Red and black broken curves: $\mathrm{NF}_{D D 1, \ell}$ and $\mathrm{NF}_{D D 1, p}$, respectively, when $n_{0}=100$. Red and black solid curves: $\mathrm{NF}_{D D 2, \ell}$ and $\mathrm{NF}_{D D 2, p}$, respectively. Red and black dash-dotted curves: $2 n_{s p, \ell}$ and $2 n_{s p, p}$, respectively.

which is the same as Eq. (68). Thus, in the high-gain region, the NFs approach $2 n_{s p, \ell}$ and $2 n_{s p, p}$ when the laser amplifier and phase-insensitive OPA are employed, respectively, and the lower limit of the NFs is $2(3 \mathrm{~dB})$.

Coherent receivers usually measure the real and imaginary parts of the signal electric field simultaneously [3]. This requirement induces a 3 -dB loss for each of the real-part and imaginary-part branches of the optical circuit after amplification. In this case, the NFs given by Eqs. (69) and (70) are modified into

$$
\begin{aligned}
\mathrm{NF}_{c o h 2, \ell} & =\frac{2}{G_{\ell} \Gamma}+\frac{2\left(G_{\ell} \Gamma-1\right) n_{s p, \ell}}{G_{\ell} \Gamma}, \\
\mathrm{NF}_{c o h 2, p} & =\frac{2}{G_{p} \Gamma}+\frac{2\left(G_{p} \Gamma-1\right) n_{s p, p}}{G_{p} \Gamma},
\end{aligned}
$$

which are close to $\mathrm{NF}_{c o h 1, \ell}$ and $\mathrm{NF}_{c o h 1, p}$, respectively, in the high-gain region.

When we employ phase-sensitive OPAs and measure only the real part of the signal electric field by using an optical phase-locked loop homodyne receiver [3], the NF is given from Eq. (61) as

$$
\mathrm{NF}_{c o h, d}=\frac{1}{G_{d} \Gamma}+\frac{\left(G_{d} \Gamma-1\right) n_{s p, d}}{G_{d} \Gamma} .
$$

This approaches $n_{s p, d}$ when $G_{d} \Gamma \gg 1$. Therefore, its lower limit is $1(0 \mathrm{~dB})$, which implies that noise-free optical amplification can be achieved.

\subsection{Numerical results on NF in coherent systems}

Figures 4, 5, and 6 show the NFs of the three types of amplifiers used in coherent systems, calculated as a function of gross gain when $\Gamma=-3 \mathrm{~dB}, \Gamma=-5 \mathrm{~dB}$, and $\Gamma=-10 \mathrm{~dB}$, respectively. The red and black solid curves represent $\mathrm{NF}_{c o h 2, \ell}$ and $\mathrm{NF}_{c o h 2, p}$, respectively, where we assume simultaneous measurements of the real and imaginary parts of the signal electric field. By contrast, the blue solid curves represent $\mathrm{NF}_{c o h, d}$ obtained by the phase-sensitive OPA. Moreover, $2 n_{s p, \ell}, 2 n_{s p, p}$, and $n_{s p, d}$ are plotted by red, black, and blue dash-dotted curves, respectively, when the gross gain is larger than $1 / \Gamma$. Note that these parameters are effective only for NF in the high-gain region. Figures 4,5 , and 6 show how the noise characteristics of the laser amplifier and OPAs in coherent systems are deteriorated by their internal loss. 


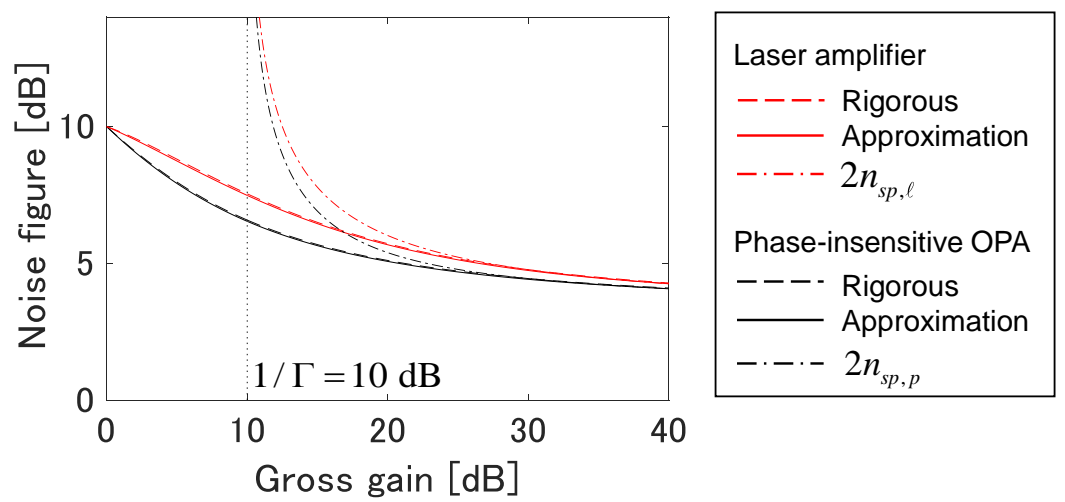

Figure 3: NFs of laser amplifiers and phase-insensitive OPAs in DD systems, calculated as a function of gross gain when $\Gamma=-10 \mathrm{~dB}$. Red and black broken curves: $\mathrm{NF}_{D D 1, \ell}$ and $\mathrm{NF}_{D D 1, p}$, respectively, when $n_{0}=100$. Red and black solid curves: $\mathrm{NF}_{D D 2, \ell}$ and $\mathrm{NF}_{D D 2, p}$, respectively. Red and black dash-dotted curves: $2 n_{s p, \ell}$ and $2 n_{s p, p}$, respectively.

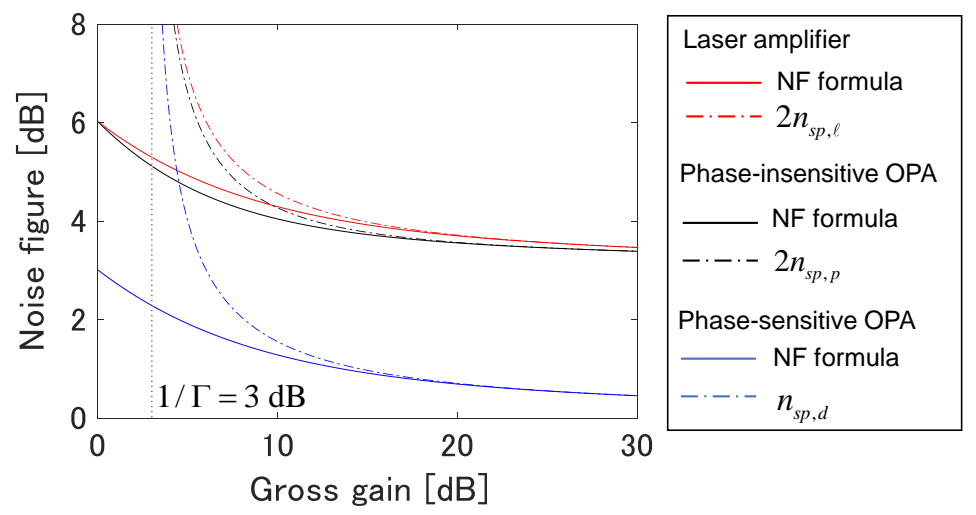

Figure 4: NFs of laser amplifiers, phase-insensitive OPAs, and phase-insensitive OPAs in coherent systems, calculated as a function of gross gain when $\Gamma=-3 \mathrm{~dB}$. Red, black, and blue solid curves: NFs of laser amplifiers, phase-insensitive OPAs, and phase-sensitive OPAs, respectively. Red, black, and blue dash-dotted curves: $2 n_{s p, \ell}, 2 n_{s p, p}$, and $n_{s p, d}$, respectively,

\section{Conclusions}

We presented a theory of noise in phase-insensitive and phase-sensitive OPAs based on the quantummechanical Langevin equations. Through derivation of theoretical expressions for the spontaneous emission factor, we analyzed the effect of the internal loss of OPAs on their noise characteristics. To the best of our knowledge, this is the first study that derives theoretical expressions for the spontaneous emission factor of OPAs. In addition, we analyzed the NFs of the laser amplifier, phase-insensitive OPA, and phase-sensitive OPA. The NFs in the DD and coherent systems were compared, and the low-noise characteristics of OPAs were theoretically demonstrated. 


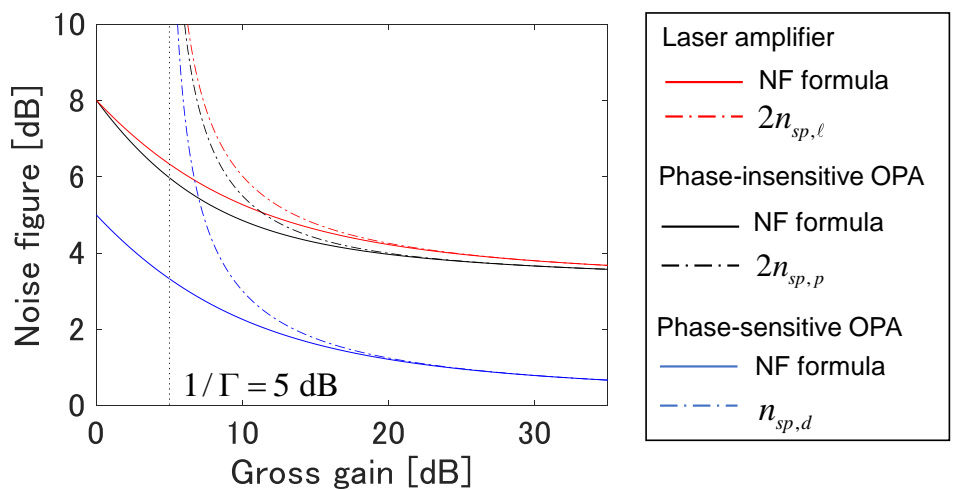

Figure 5: NFs of laser amplifiers, phase-insensitive OPAs, and phase-insensitive OPAs in coherent systems, calculated as a function of gross gain when $\Gamma=-5 \mathrm{~dB}$. Red, black, and blue solid curves: NFs of laser amplifiers, phase-insensitive OPAs, and phase-sensitive OPAs, respectively. Red, black, and blue dash-dotted curves: $2 n_{s p, \ell}, 2 n_{s p, p}$, and $n_{s p, d}$, respectively,

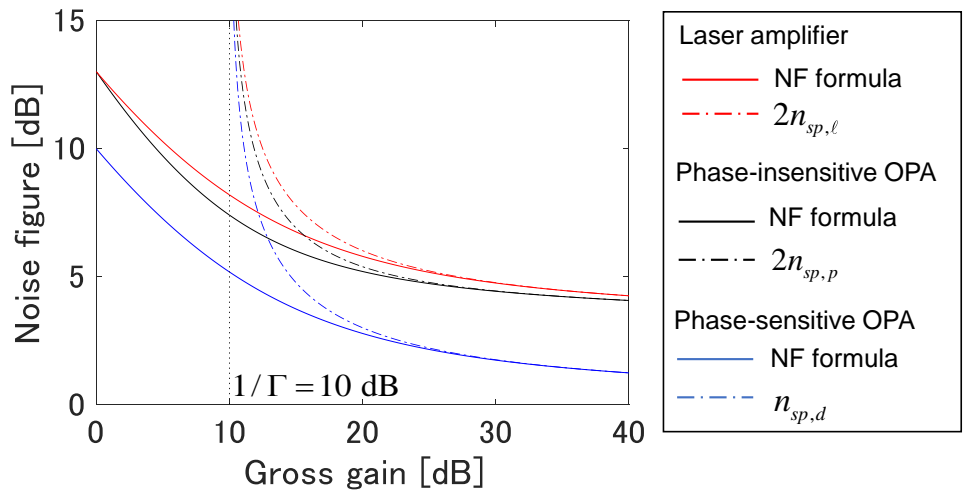

Figure 6: NFs of laser amplifiers, phase-insensitive OPAs, and phase-insensitive OPAs in coherent systems, calculated as a function of gross gain when $\Gamma=-10 \mathrm{~dB}$. Red, black, and blue solid curves: NFs of laser amplifiers, phase-insensitive OPAs, and phase-sensitive OPAs, respectively. Red, black, and blue dash-dotted curves: $2 n_{s p, \ell}, 2 n_{s p, p}$, and $n_{s p, d}$, respectively,

\section{References}

[1] K. Shimoda, H. Takahashi, and C. H. Townes, "Fluctuations in amplification of quanta with application to maser amplifiers," J. Phys. Soc. Jpn., vol. 12, no. 6, pp. 686-700, 1957.

[2] T. Kobayashi, S. Shimizu, M. Nakamura, T. Umeki, T. Kazama, R. Kasahara, F. Hamaoka, M. Nagatani, H. Yamazaki, T. Mizuno, H. Nosaka, and Y. Miyamoto, "Wideband inline-amplified WDM transmission using PPLN-based OPA with over-10-THz bandwidth," in Optical Fiber Communication Conference, OSA Technical Digest (Optical Society of America, 2020), Th4C.7.

[3] K. Kikuchi, "Fundamentals of coherent optical fiber communications," J. Lightwave Technol., vol. 34, no. 1, pp. 157-179, 2016.

[4] K. Kikuchi, "Theory of spontaneous emission factors of optical parametric amplifiers," in Optical Fiber Communication Conference, OSA Technical Digest (Optical Society of America, 2021), W6A.38. 
[5] M. O. Scully and M. S. Zubairy, Quantum Optics. Cambridge University Press, 1997.

[6] H. A. Haus, Electromagnetic Noise and Quantum Optical Measurements. Springer, 2000.

[7] S. Stenholm, "The theory of quantum amplifiers," Physica Scripta, vol. T12, pp. 56-66, 1986.

[8] T. Okoshi and K. Kikuchi, Coherent Optical Communication Systems. Springer, 1988.

[9] A. Yariv, Quantum Electronics (Third Edition). John Wiley \& Sons, Inc., 1989. 